\title{
MONOTONE AND OPEN MAPPINGS ON MANIFOLDS. I
}

\author{
BY \\ JOHN J. WALSH
}

\begin{abstract}
Sufficient conditions are given for the existence of open mappings from a p. 1. manifold $M^{m}, m \geqslant 3$, onto a polyhedron $Q$. In addition, it is shown that a mapping $f$ from $M^{m}, m \geqslant 3$, to $Q$ is homotopic to a monotone mapping of $M$ onto $Q$ iff $f_{*}: \pi_{1}(M) \rightarrow \pi_{1}(Q)$ is onto. Finally, it is shown that a monotone mapping of $M^{m}, m \geqslant 3$, onto $Q$ can be approximated by a monotone open mapping of $M$ onto $Q$.
\end{abstract}

In a recent paper [19], D. Wilson constructs monotone open mappings of any compact, connected p.1. manifold $M^{m}, m \geqslant 3$, onto any cell. In addition, he constructs light open mappings of three dimensional manifolds onto $n$ cells for $n \geqslant 3$. The question of the existence of such mappings appears in a list of 45 problems compiled by Eilenberg in 1949 in [3]. He asks whether there is an open mapping of a manifold onto a space of higher dimension and whether there is a light open mapping of a manifold with each point inverse a Cantor set. The answer to the first question was given by R. D. Anderson in [1] where he announced the existence of monotone open mappings of any p.l. manifold $M^{m}, m \geqslant 3$, onto any cell; however, he never published a proof. Results similar to Anderson's were obtained by Keldys at about the same time in [7] , [8], and [9]. The recent work of Wilson in [19] answers the second question.

In contrast, for two dimensional manifolds R. L. Moore [13] and Roberts and Steenrod [14] show that monotone images have dimension at most two and Stoilow [16] and G. T. Whyburn ([4] is an excellent survey of his work) show that light open mappings are branched coverings and, hence, do not raise dimension.

In this paper, we begin a systematic study of the existence of monotone, monotone open, open, and light open mappings from manifolds to polyhedra. In particular, we are interested in when a mapping "is homotopic to" or "can be approximated by" one of the above four types of mappings. In $\$ 2$, we completely determine those mappings homotopic to monotone mappings. In $\S 3$, we give general criteria for the existence of open mappings and use this to show that monotone mappings can be approximated by monotone open mappings; this leads to a determination of those mappings homotopic to monotone open mappings. In a

Received by the editors April 15, 1974 and, in revised form, July 1, 1974. AMS (MOS) subject classifications (1970). Primary 54C10; Secondary 57C99.

Key words and phrases. Monotone mapping, open mapping, p. 1. manifold, polyhedron. Copyright @ 1975, American Mathematical Society 
second paper, we will completely determine those mappings homotopic to open mappings and we will approximate open mappings by light open mappings, this leading to a determination of those mappings homotopic to light open mappings. In a third paper, we will investigate to what extent the assumption that the image be a polyhedron can be weakened. A note of caution, in dealing with light open mappings we are faced with the fact that light mappings cannot lower dimension; hence, all of the results on light open mappings will include the assumption that the image be a p.l. manifold of dimension at least as large as that of the domain.

The basic techniques used throughout are similar to those used by D. Wilson in [18], [19], and [20]; indeed, his work in [18] is an excellent starting point. Also, $\S 1$ consists in part of redoing some of the work in [20, $\S 1-4]$.

This paper forms a portion of the author's dissertation written under the direction of L. F. McAuley at the State University of New York at Binghamton.

Terminology. We will assume that manifolds and polyhedra are compact but manifolds may have boundary; p.1. = piecewise linear. Polyhedra will be viewed as subsets of some Euclidean space; the simplices of a simplicial complex triangulating a polyhedron will be considered to be subsets of the polyhedron (i.e., not abstract objects). If $K$ is a simplicial complex, then $K^{i}$ will denote the $i$ th skeleton of $K, t(K)$ will be all top dimensional simplices of $K$ (i.e., $\sigma \in$ $t(K)$ iff $\sigma<\tau \Rightarrow \sigma=\tau) ; \beta^{n} K$ will denote the $n$th barycentric subdivision of $K$. A collection $A$ of nonempty closed subsets of a space $X$ is simple provided: for $a, a^{\prime} \in A$, (i) if $a \cap a^{\prime} \neq \varnothing$, then $\operatorname{int}\left(a \cup a^{\prime}\right)$ is a dense connected subset of $a \cup a^{\prime}$, and (ii) if $a \neq a^{\prime}$, then $\operatorname{int}(a) \cap \operatorname{int}\left(a^{\prime}\right)=\varnothing$. Int( ), bd( ), and $\operatorname{cl}()$ will denote the topological interior, boundary, and closure, respectively. The boundary of a manifold $M$ will be denoted $\partial M$.

If $A$ is a collection of sets, then $A^{*}$ is the union of the sets in $A$. Let st $^{0}(b, A)=\{a \in A \mid a \subseteq b\}$, let st ${ }^{1}(b, A)=\{a \in A \mid a \cap b \neq \varnothing\}$, and, inductively, let $\mathrm{st}^{i}(b, A)=\mathrm{st}^{1}\left(\mathrm{st}^{i-1}(b, A)^{*}, A\right), i \geqslant 2$. At times $\mathrm{st}(b, A)$ will be used for $\mathrm{st}^{1}(b, A)$. If $K$ is a triangulation of $M^{m}$ (manifold of dimension $m$ ) and $L$ is a subcomplex of $K$ and $A$ is a subset of $t(K)$, then let $\operatorname{ST}^{0}(L, A)=\{\sigma \in A \mid \sigma \subseteq L\}$, let $\mathrm{ST}^{1}(L, A)=\{\sigma \in A \mid \sigma$ meets $L$ in an $m$ or $m-1$ face $\}$, and, inductively, let $\mathrm{ST}^{i}(L, A)=\mathrm{ST}^{1}\left(\mathrm{ST}^{i-1}(L, A)^{*}, A\right), i \geqslant 2$.

By a mapping we will mean a continuous function. A mapping is monotone (resp., light) provided each point inverse is connected (resp., totally disconnected). A mapping is open if the image of each open set is open. The mesh of a collection of sets is the supremum of their diameters. Let $I$ denote the unit interval, let $D^{n}$ denote a p.1. $n$ ball, let $\dot{D}^{n}$ denote its boundary, and let $1 / 2 D^{n}$ denote a p.1. $n$ ball in $D^{n}-\dot{D}^{n}$. 
Definition. If $K$ is a triangulation of some polyhedron and $\bar{K}$ is a subcomplex of $K$, then $(K, \bar{K})$ is an acceptable pair of dimension $m$ provided $K-\bar{K}$ is an $m$ dimensional manifold, possibly with boundary, and $\bar{K}$ is a union of $m-1$ simplices each of which is a face of exactly one $m$ simplex of $K$. Denote the pair $(K, \varnothing)$ by $K$.

1. This section contains the basic technique used in the various constructions throughout this paper. The presentation in this section is a variation of that of D. Wilson in $[20, \S \S 1-4]$.

(1.0) Convention. Let $(K, \bar{K})$ be an acceptable pair of dimension $m$ and let $P$ be a collection of nonempty sets with $P^{*}=K$, with each set in $P$ a union of sets in $t(K)$, and with different sets in $P$ having disjoint interiors. If $p, p^{\prime} \in P$ and $A$ is a subset of $K$, then the statement connect $p$ to $p^{\prime}$ with a tube in $A$ means the following. Let $B$ be a polyhedral tubular neighborhood of a polyhedral arc with $B \subseteq \operatorname{int}(A) \cap(K-\bar{K})$ and with each of $B \cap p$ and $B \cap p^{\prime}$ a face of $B$ (here we are considering $B$ to be homeomorphic to $D^{m-1} \times I$ with faces $D^{m-1} \times\{0\}$ and $\left.D^{m-1} \times\{1\}\right)$. Alter the collection $P$ by adding $B$ to $p$ or $p^{\prime}$ (unless it is stated to add part to $p$ and part to $p^{\prime}$ ) and replace each $p^{\prime \prime} \in P-\left\{p, p^{\prime}\right\}$ with $\operatorname{cl}\left(p^{\prime \prime}-B\right)$. In general, we shall continue to call the altered collection $P$.

(1.1) Let $(K, \bar{K})$ and $L$ be acceptable pairs and let $g: \bar{K} \rightarrow L$ be a mapping which linearly maps each simplex of $\bar{K}$ into a simplex of $L$. Let $P$ be a collection of nonempty sets with $P^{*}=K$, with each set in $P$ a union of sets in $t(K)$, and with different sets in $P$ having disjoint interiors. Let $T: P \rightarrow t(L)$ be a one-to-one and onto function satisfying: $p_{q} \in P$.

(1.1.1) $T\left(p_{1}\right) \cap \cdots \cap T\left(p_{q}\right) \neq \varnothing$ whenever $p_{1} \cap \cdots \cap p_{q} \neq \varnothing ; p_{1}, \ldots$,

(1.1.2) If $\sigma \in \bar{K}$ and $\sigma \subseteq p \in P$, then $g(\sigma) \subseteq T(p)$.

Extend $g$ to all of $K$ as follows. If $v$ is a vertex of $\beta^{1} K$ and $v \notin \bar{K}$, then let $g(v)=$ barycenter of $\bigcap\{T(p) \mid v \in p\}$; condition (1.1.1) guarantees that this makes sense. It follows from conditions (1.1.1) and (1.1.2) that $g$ extends linearly to each simplex of $\beta^{1} K$. By taking a subdivision of $\beta^{1} K$, say $K^{\prime}$, and by adjusting $g$ on int $(\sigma)$ for various $\sigma \in t(K)$, we can assume that $g$ linearly maps each simplex of $K^{\prime}$ into a simplex of $\beta^{1} L$ and that if $\sigma \in t(K), \sigma \subseteq p \in P$, then $g(\sigma) \cap \operatorname{int}(\tau) \neq \varnothing$ for each $\tau \subseteq T(p)$, $\tau \in t\left(\beta^{1} L\right)$.

Define a new triple $T^{\prime}: P^{\prime} \rightarrow t\left(\beta^{1} L\right)$ as follows: for each $\sigma \in t\left(K^{\prime}\right)$, let $\hat{T}(\sigma)$ be any $\tau \in t\left(\beta^{1} L\right)$ with $g(\sigma) \subseteq \tau$; let $P^{\prime}=\left\{\hat{T}^{-1}(\tau)^{*} \mid \tau \in t\left(\beta^{1} L\right)\right\}$; and let $T^{\prime}\left(\hat{T}^{-1}(\tau)^{*}\right)=\tau$. It follows directly that $T^{\prime}$ is one-to-one and onto and that, upon replacing $T, P, K$, and $t(L)$ with $T^{\prime}, P^{\prime}, K^{\prime}$, and $t\left(\beta^{1} L\right)$, respectively, conditions (1.1.1) and (1.1.2) are satisfied. 
(1.2) REMARK. In addition to the method outlined above, we shall have cause to alter certain triples $T: P \rightarrow t(L)$ by altering the sets in $P$. At all times, it will be necessary to have condition (1.1.1) satisfied. However, no attempt will be made to explicitly verify condition (1.1.1) after each alteration; such verification is usually immediate from the construction. Indeed, at times, the complex. ity of a construction results from the need to maintain condition (1.1.1).

(1.3) Basic construction. Let $(K, \bar{K})$ be an acceptable pair of dimension $m$ $(2)$ and let $L$ be a finite simplicial complex. Let $g: \bar{K} \rightarrow L$ and $P$ be as in (1.1) and let $T: P \rightarrow t(L)$ be a one-to-one and onto function satisfying conditions (1.1.1) and (1.1.2). By running tubes in various of the sets int(st $\left.\left(v, \beta^{2} K\right)^{*}\right)$ $\cap(K-\bar{K})$, where $v$ is a vertex of $\beta^{1} K$, the collection $P$ can be altered so that the collection of components of the sets in $P$ is simple. Call the altered collection $P_{0}$ and the induced function $T_{0}$; let $K_{0}$ be a subdivision of $K$ with each set in $P_{0}$ a union of sets in $t\left(K_{0}\right)$. Of course, $L$ remains unchanged.

Let $T^{\prime}: P^{\prime} \rightarrow t\left(\beta^{1} L\right)$ be a triple obtained from $T_{0}: P_{0} \rightarrow t(L)$ by the method in (1.1); observe that if $p^{\prime} \in P^{\prime}$ and $p \in P$ with $p^{\prime} \subseteq p$, then $p^{\prime}$ meets the interior of each component of $p$. Make the following alterations, in order, to the collection $P^{\prime}$.

(1.3.1) For each $p \in P_{0}$ and for each pair $\sigma_{1}, \sigma_{2} \in t\left(K_{0}\right)$ with $\sigma_{1} \cup \sigma_{2} \subseteq p$ and $\sigma_{1} \cap \sigma_{2}$ an $m$ or $m-1$ face, run tubes in $\operatorname{int}\left(\sigma_{1} \cup \sigma_{2}\right) \cap\left(K^{\prime}-\bar{K}^{\prime}\right)$ so that if $p_{1}^{\prime}, p_{2}^{\prime} \in P^{\prime}$ with $p_{1}^{\prime} \cup p_{2}^{\prime} \subseteq p$, then the $\operatorname{int}\left(p_{1}^{\prime} \cup p_{2}^{\prime}\right) \cap\left(\sigma_{1} \cup \sigma_{2}\right)$ is connected (include the case that $p_{1}^{\prime}=p_{2}^{\prime}$ ). Since $\bigcap\left\{\tau \mid \tau \in t\left(\beta^{1} L\right)\right.$ and $\left.\tau \subseteq T_{0}(p)\right\} \neq \varnothing$, condition (1.1.1) will not be violated.

(1.3.2) For each pair $p_{1}, p_{2} \in P_{0}$ with $p_{1} \cap p_{2} \neq \varnothing$, but $p_{1} \neq p_{2}$, and for each pair $\sigma_{1}, \sigma_{2} \in t\left(K_{0}\right)$ with $\sigma_{i} \subseteq p_{i}, i=1,2$, and with $\sigma_{1} \cap \sigma_{2}$ an $m-1$ simplex make the following alterations. Let $p_{1}^{\prime}, p_{2}^{\prime} \in P^{\prime}$ with $p_{i}^{\prime} \subseteq p_{i}, i=1,2$, with $p_{1}^{\prime} \cap p_{2}^{\prime}$ containing an $m-1$ simplex of $K^{\prime}$, and with $p_{1}^{\prime} \cap p_{2}^{\prime}$ containing the barycenter of $\sigma_{1} \cap \sigma_{2}$. (It follows that $T^{\prime}\left(p_{1}^{\prime}\right) \cap T^{\prime}\left(p_{2}^{\prime}\right)$ contains the barycenter of $T_{0}\left(p_{1}\right) \cap T_{0}\left(p_{2}\right)$; hence the following alteration will not violate condition (1.1.1).) For each pair $\tau_{1}, \tau_{2} \in t\left(\beta^{1} L\right)$ with $\tau_{i} \subseteq T_{0}\left(p_{i}\right)$ and with $\tau_{i}$ containing the barycenter of $T_{0}\left(p_{1}\right) \cap T_{0}\left(p_{2}\right), i=1,2$, connect $\operatorname{int}\left(T^{\prime-1}\left(\tau_{1}\right)\right) \cap \sigma_{1}$ to $\operatorname{int}\left(T^{\prime-1}\left(\tau_{2}\right)\right) \cap \sigma_{2}$ with a tube in int $\left(\sigma_{1} \cup \sigma_{2}\right) \cap \operatorname{int}\left(T^{\prime-1}\left(\tau_{1}\right) \cup p_{1}^{\prime} \cup p_{2}^{\prime} \cup T^{\prime-1}\left(\tau_{2}\right)\right)$, adding the part of the tube in $\sigma_{i}$ to $T^{\prime-1}\left(\tau_{i}\right), i=1,2$.

(1.3.3) For each pair $p_{1}, p_{2} \in P_{0}$ with $p_{1} \cap p_{2} \neq \varnothing$, but $p_{1} \neq p_{2}$, and for each pair $\sigma_{1}, \sigma_{2} \in t\left(K_{0}\right)$ with $\sigma_{i} \subseteq p_{i}, i=1,2$, and with $\sigma_{1} \cap \sigma_{2}$ an $m-1$ simplex make the following alterations. If $\tau_{1}, \tau_{2} \in t\left(\beta^{1} L\right)$ with $\tau_{i} \subseteq T_{0}\left(p_{i}\right), i=$ 1,2 , with $\tau_{1} \cap \tau_{2} \neq \varnothing$, but with $\tau_{1} \cap \tau_{2}$ not containing the barycenter of $T_{0}\left(p_{1}\right) \cap T_{0}\left(p_{2}\right)$, let $\rho_{i} \in t\left(\beta^{1} L\right)$ with $\rho_{i} \subseteq T_{0}\left(p_{i}\right), i=1,2$, with $\tau_{1} \cap \tau_{2} \subseteq \rho_{1}$ $\cap \rho_{2}$, and with $\rho_{1} \cap \rho_{2}$ containing the barycenter of $T_{0}\left(p_{1}\right) \cap T_{0}\left(p_{2}\right)$. Connect 
$\operatorname{int}\left(T^{\prime-1}\left(\tau_{1}\right)\right) \cap \sigma_{1}$ to $\operatorname{int}\left(T^{\prime-1}\left(\tau_{2}\right)\right) \cap \sigma_{2}$ with a tube in $\operatorname{int}\left(\sigma_{1} \cup \sigma_{2}\right) \cap$ $\operatorname{int}\left(T^{\prime-1}\left(\tau_{1}\right) \cup T^{\prime-1}\left(\rho_{1}\right) \cup T^{\prime-1}\left(\rho_{2}\right) \cup T^{\prime-1}\left(\tau_{2}\right)\right)$, adding the part of the tube in $\sigma_{i}$ to $T^{\prime-1}\left(\tau_{i}\right), i=1,2$.

Let $T_{1}: P_{1} \rightarrow t\left(\beta^{1} L\right)$ be the triple obtained from $T^{\prime}: P^{\prime} \rightarrow t\left(\beta^{1} L\right)$ after all the above alterations have been made and let $K_{1}$ be a subdivision of $K^{\prime}$ such that each set in $P_{1}$ is a union of sets in $t\left(K_{1}\right)$. This new triple satisfies the appropriate reformulation of conditions (1.1.1) and (1.1.2). The collection of components of the sets in $P_{1}$ is simple. If $\tau_{i} \in t\left(\beta^{1} L\right)$ and $\sigma_{i} \in t(L)$ with $\tau_{i} \subseteq \sigma_{i}, i=$ 1,2 , then $T_{1}^{-1}\left(\tau_{1}\right) \cup T_{1}^{-1}\left(\tau_{2}\right)$ has exactly the same number of components as $T_{0}^{-1}\left(\sigma_{1}\right) \cup T_{0}^{-1}\left(\sigma_{2}\right)$, including the cases where $\sigma_{1}=\sigma_{2}$ or $\tau_{1}=\tau_{2}$.

(1.4) Proposition. Let $(K, \bar{K})$ be an acceptable pair of dimension at least 3 and let $L$ be a finite simplicial complex. Let $g: \bar{K} \rightarrow L$ and $P$ be as in $(1.1)$ and let $T: P \rightarrow t(L)$ be a one-to-one and onto function satisfying conditions (1.1.1) and (1.1.2). Then there exists a mapping $f$ from $K$ onto $L$ with $f=$ $g$ on $\bar{K}$, with $f(p) \subseteq \operatorname{st}(T(p), L)^{*}$ for each $p \in P$, and with $f^{-1}(y)$ having the same number of components as $\{p \in P \mid y \in T(p)\}^{*}$.

Proof. Let $\left\{T_{n}: P_{n} \rightarrow t\left(\beta^{n} L\right)\right\}$ be a sequence of triples obtained by repeated applications of (1.3). Define $f: K \rightarrow L$ by $f\left(\bigcap_{i=0}^{\infty} p_{i}\right)=\bigcap_{i=0}^{\infty} T_{i}\left(p_{i}\right)$ for each nested sequence $\left\{p_{0} \supseteq p_{1} \supseteq \cdots \mid p_{i} \in P_{i}\right\}$. It is a direct verification to show that $f$ has the desired properties.

(1.4.1) COROLlary. In addition to the hypothesis of (1.4), assume that each of the sets in $P$ is connected and that $T^{-1}\left(\sigma_{1}\right) \cap T^{-1}\left(\sigma_{2}\right) \neq \varnothing$ whenever $\sigma_{1} \cap \sigma_{2} \neq \varnothing, \sigma_{1}, \sigma_{2} \in t(L)$. Then the mapping $f$ constructed is monotone.

If we assume $W=\varnothing$, then the next corollary is due to D. Wilson [20]; indeed, the machinery developed in this section is, in essence, his proof.

(1.4.2) Corollary. Let $M^{m}$ be a connected, p. l. manifold with $m \geqslant 3$ and let $\Delta^{n}$ be an $n$ simplex with $n \geqslant 0$. Let $K$ be a triangulation of $M$ with $W$ a subcomplex of $K$ and with $W \subseteq \partial M$ (we permit $W=\varnothing$ and/or $\partial M=\varnothing$ ). Let $g: W \rightarrow \Delta^{n}$ be a mapping which linearly maps each simplex of $K$ in $W$ into a simplex of $\Delta^{n}$. Then $g$ extends to a monotone mapping of $M$ onto $\Delta^{n}$.

Proof. Extend $g$ to a mapping of all of $\partial M$ so that it still maps simplices linearly into simplices. Let $(K, \bar{K})=(M, \partial M), L=\Delta^{n}, P=\{M\}$, and $T(M)=$ $\Delta^{n}$; the result now follows from (1.4.1).

The proof of the following corollary illustrates D. Wilson's approach in [22] to constructing monotone mappings of various degrees between spheres. The corollary is also a direct consequence of Theorem (2.0) in the next section. 
(1.4.3) CoROllaRY. If $m \geqslant 3$, then there are monotone mappings of any degree from $S^{m}$ onto itself.

Proof. Let $g: \partial \Delta^{m} \rightarrow \partial \Delta^{m}$ be a piecewise linear mapping of degree $q$; use (1.4.2) to extend $g$ to a monotone mapping of $\Delta^{m}$ onto $\Delta^{m}$. Consider $S^{m}$ as the double of $\Delta^{m}$ and use $f$ on each half to obtain a monotone mapping of $S^{m}$ onto itself. (The mapping $f$ will be monotone if $g: \partial \Delta^{m} \rightarrow \partial \Delta^{m}$ is onto; this is always the case if $q \neq 0$ and can be arranged if $q=0$.) It is an exercise in using the exact sequences of homology (in particular, the Mayer-Vietoris sequence) to show that this mapping has degree $q$.

2. This section contains a proof of the following theorem.

(2.0) THEOREM. A mapping f from a compact and connected p.l. manifold $M^{m}(m \geqslant 3)$ to a connected polyhedron $Q$ is homotopic to a monotone mapping of $M$ onto $Q$ if and only if $f_{*}: \pi_{1}(M) \rightarrow \pi_{1}(Q)$ is surjective.

The "only if" half of this theorem is easily proved and is well known [15]. The proof of the other half is broken into two parts. First, a method is developed in Proposition (2.1) for producing a triple, $T: P \rightarrow t(L)$, as in Corollary (1.4.1), from a continuous function. Second, this method is carefully analyzed in order to produce the desired homotopy.

(2.1) Proposition. Assume the hypothesis of Theorem (2.0). Then there exist triangulations $K$ and $L$ of $M$ and $Q$, respectively, and there exists a triple $T$ : $P \rightarrow t(L)$ as in Corollary (1.4.1).

Proof. Let $\hat{K}$ and $L$ be triangulations of $M$ and $Q$, respectively, and let $F_{1}$ be a mapping satisfying:

(2.1.1) $F_{1}$ is homotopic to $f$.

(2.1.2) $F_{1}$ linearly maps each simplex of $\hat{K}$ into a simplex of $L$.

(2.1.3) The image of $F_{1}$ contains the barycenter of each simplex of $t(L)$.

(2.2) For each $\sigma \in t(\hat{K})$, let $\bar{T}(\sigma)$ be some element of $t(L)$ containing $F_{1}(\sigma)$. Let $\hat{P}=\left\{\bar{T}^{-1}(\tau)^{*} \mid \tau \in t(L)\right\}$ and define $\hat{T}: \hat{P} \rightarrow t(L)$ by $\hat{T}\left(\bar{T}^{-1}(\tau)^{*}\right)=\tau$. Observe that $\hat{T}\left(p_{1}\right) \cap \cdots \cap \hat{T}\left(p_{q}\right) \neq \varnothing$ whenever $p_{1} \cap \cdots \cap p_{q} \neq \varnothing, p_{i} \in \hat{P}$. Use the method of (1.3) to alter the collection $\hat{P}$ so that the collection of components of the sets in $\hat{P}$ is simple. Continue to denote this triple by $\hat{T}: \hat{P} \rightarrow t(L)$ and continue to denote by $\hat{K}$ a triangulation "refining" the sets in $\hat{P}$.

(2.3) Let $C_{1}, C_{2}$ be components of $p_{1}, p_{2} \in \hat{P}$, respectively, where $\hat{T}\left(p_{1}\right) \cap$ $\hat{T}\left(p_{2}\right) \neq \varnothing$ but $C_{1} \cap C_{2}=\varnothing$. Let $\alpha: I \rightarrow\left(\hat{K}-\hat{K}^{m-2}\right)$ be a p.l. embedding with $\alpha(I)=\bigcup_{i=1}^{r} J_{i}$ where each $J_{i}$ is a spanning arc of a $\sigma_{i} \in t(\hat{K}), J_{i} \cap J_{i+1}$ is a point, $\alpha(0) \in C_{1}-\operatorname{int}\left(C_{1}\right)$, and $\alpha(1) \in C_{2}-\operatorname{int}\left(C_{2}\right)$. Let $\sigma_{0}$ (resp. $\sigma_{r+1}$ ) be an 
element of $t(\hat{K})$ contained in $p_{1}$ (resp. $p_{2}$ ) with $\alpha(0)$ (resp. $\alpha(1)$ ) contained in $\operatorname{bd}\left(\sigma_{0}\right)$ (resp. bd $\left.\left(\sigma_{r+1}\right)\right)$. Write $\dot{D}^{2}=\bigcup_{i=0}^{r} H_{i}$, a union of arcs with successive arcs meeting in a point. For each $\sigma_{i}, i=0, \ldots, r+1$, let $\hat{T}\left(\sigma_{i}\right)=\hat{T}(p)$ where $\sigma_{i} \subseteq p \in \hat{P}$; recall that $\hat{T}\left(\sigma_{i}\right) \cap \hat{T}\left(\sigma_{i+1}\right) \neq \varnothing$. Define a p.1. map $\Omega_{\alpha}: \dot{D}^{2} \rightarrow Q$ as follows: if $\hat{T}\left(\sigma_{i}\right) \neq \hat{T}\left(\sigma_{i+1}\right)=\cdots=\hat{T}\left(\sigma_{i+j}\right) \neq \hat{T}\left(\sigma_{i+j+1}\right)$, then $\Omega_{\alpha}$ embeds $H_{i+1} \cup \cdots \cup H_{i+j}$ as a spanning arc of $\hat{T}\left(\sigma_{i+1}\right)$, spanning from the barycenter of $\hat{T}\left(\sigma_{i}\right) \cap \hat{T}\left(\sigma_{i+1}\right)$ to the barycenter of $\hat{T}\left(\sigma_{i+1}\right) \cap \hat{T}\left(\sigma_{i+j+1}\right)$. Finally, $\Omega_{\alpha}\left(H_{0}\right)$ is to be a spanning arc of $\hat{T}\left(p_{1}\right) \cup \hat{T}\left(p_{2}\right)$.

(2.4) Lemma. An arc $\alpha^{\prime}$ can be chosen so that $\Omega_{\alpha^{\prime}}$ extends to a mapping of $D^{2}$ into $Q$.

Proof. By adjusting $F_{1}$ on int $\left(\sigma_{1}\right)$, assume that $F_{1}\left(\operatorname{int}\left(\sigma_{1}\right)\right) \subseteq \operatorname{int}\left(\hat{T}\left(\sigma_{1}\right)\right)$ and that, for some $x_{0} \in \operatorname{int}\left(\sigma_{1}\right), y_{0}=F_{1}\left(x_{0}\right) \in \Omega_{\alpha}\left(H_{1}\right)$. Let $\gamma: \dot{D}^{2} \rightarrow$ $\left(\hat{K}-\hat{K}^{m-2}\right)$ be a p.l. embedding with $\gamma\left(\dot{D}^{2}\right) \cap \alpha(I)=\left\{x_{0}\right\}$ and with $F_{1} \circ \gamma$ equal to $\Omega_{\alpha}$ in $\pi_{1}\left(Q, y_{0}\right)$; this is the only point at which we use the assumption that $f_{*}$, and hence $\left(F_{1}\right)_{*}$, is a surjection. Observe the $\left(F_{1} \circ \gamma\right)^{-1} * \Omega_{\alpha}$ is zero in $\pi_{1}\left(Q, y_{0}\right)$. Let $\alpha^{\prime}$ be the arc obtained by moving along $\alpha$ to $x_{0}$, then moving "backwards" along $\gamma$, and then moving along the remainder of $\alpha$; by a slight adjustment, remove the singularity at $x_{0}$.

(2.5) Let $U=\left\{\left\{C_{1}, C_{2}\right\} \mid C_{i}\right.$ is a component of $p_{i}, i=1,2, C_{1} \cap C_{2}=\varnothing$, and $\left.\hat{T}\left(p_{1}\right) \cap \hat{T}\left(p_{2}\right) \neq \varnothing\right\}$ be a collection of unordered pairs. The set $U$ consists of exactly those pairs of sets which must be "connected together" in order to have the hypothesis of Corollary (1.4.1) satisfied. For the final time, we explicitly emphasize that the condition " $\hat{T}\left(p_{1}\right) \cap \cdots \cap \hat{T}\left(p_{q}\right) \neq \varnothing$ whenever $p_{1} \cap \cdots \cap p_{q} \neq \varnothing$ " must be preserved at each stage of the following alterations. For each $u \in U$, let $\alpha_{u}$ be the arc guaranteed by (2.4) with $\alpha_{u}(I)=\bigcup_{i=1}^{r_{u}} J_{i}^{u}$ where $J_{i}^{u}$ is a spanning arc of $\sigma_{i}^{u}$. Let $\Omega_{u}$ be the mapping constructed from $\alpha_{u}$ in (2.3). Denote by $N_{u}=\alpha_{u}(I) \times D^{m-1}=\bigcup_{i=1}^{r_{u}}\left(J_{i}^{u} \times D^{m-1}\right)$ a tubular neighborhood of $\alpha_{u}$ in $M$; see Figure 1. (For convenience, we shall not distinguish between $N_{u}$ and its homeomorphic copy $\alpha_{u} \times D^{m-1}$.) Make the above choices so that the $N_{u}$ 's are

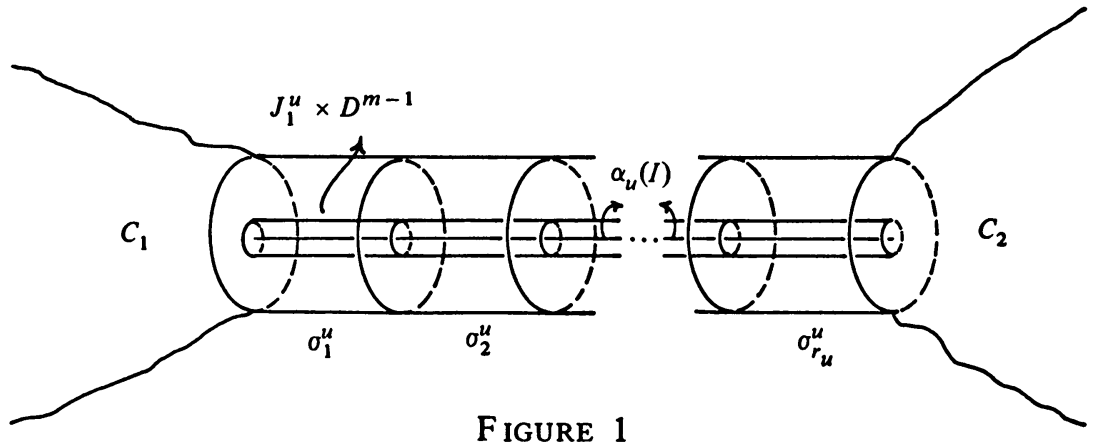

F IGURE 1 
pairwise disjoint. Let $\Gamma_{u}: D^{2} \rightarrow Q$ be an extension of $\Omega_{u}$ guaranteed by (2.4); of course, we assume that $\Gamma_{u}$ is simplicial on suitable subdivisions.

(2.6) Recall that $\Omega_{u}: \dot{D}^{2}=\bigcup_{i=0}^{r_{u}} H_{i}^{u} \rightarrow Q$. Define a p.l.mapping $g_{u}$ : $N_{u} \rightarrow D^{2}$ as follows. Let $g_{u}\left(\alpha_{u}(0) \times D^{m-1}\right)=H_{0}^{u} \cap H_{1}^{u}$ and let $g_{u}\left(\alpha_{u}(1) \times D^{m-1}\right)=H_{r_{u}}^{u} \cap H_{0}^{u}$; define $g_{u}$ on $\alpha_{u}(I) \times \dot{D}^{m-1}$ by projecting $J_{i}^{u} \times$ $\dot{D}^{m-1}$ onto $J_{i}^{u} \times\{0\}$ and sending $J_{i}^{u} \times\{0\}$ to $H_{i}^{u}$ by a homeomorphism. Let $g_{u}$ map $\alpha_{u}(I) \times\{0\}$ homeomorphically onto $H_{0}^{u}$, let $g_{u}\left(\{x\} \times 1 / 2 D^{m-1}\right)=g_{u}(\{x\} \times\{0\})$, and extend $g_{u}$ to a mapping of the rest of $N_{u}$.

Let $K^{\prime}$ be a subdivision of $\hat{K}$ such that each $\Gamma_{u} \circ g_{u}$ linearly maps each simplex of $K^{\prime}$ in $N_{u}$ into a simplex of $L$.

(2.7) Let $\hat{P}_{1}=\left\{\operatorname{cl}\left(p-\bigcup_{u \in U} N_{u}\right) \mid p \in \hat{P}\right\}$ and let $\hat{T}_{1}\left(\operatorname{cl}\left(p-\bigcup_{u \in U} N_{u}\right)\right)=$ $\hat{T}(p)$. For each $u=\left\{C_{1}, C_{2}\right\} \in U, C_{i} \subseteq p_{i}, i=1,2$, add to $\operatorname{cl}\left(p_{i}-\bigcup_{u \in U} N_{u}\right)$ the part of $\alpha_{u}(I) \times 1 / 2 D^{m-1}$ which $\Gamma_{u} \circ g_{u}$ maps into $\hat{T}\left(p_{i}\right), i=1,2$; call this collection $\hat{P}_{2}$ and let $\hat{T}_{2}$ be the induced function. Observe that the triple $\hat{T}_{2}: \hat{P}_{2} \rightarrow$ $t(L)$ satisfies all the desired properties except that $\hat{P}_{2}^{*} \neq M$. We now proceed to remedy this deficiency.

(2.8) Let $\tau \in t\left(K^{\prime}\right)$ with $\tau \nsubseteq \hat{P}_{2}^{*}$ but with $\tau \cap p$ containing an $m-1$ face for some $p \in \hat{P}_{2}$. Run a tube in $\operatorname{int}(p \cup \tau)$ connecting $\tau$ to some $p^{\prime} \in \hat{P}_{2}$ where $\Gamma_{u} \circ g_{u}(\tau) \subseteq \hat{T}_{2}\left(p^{\prime}\right)$; add $\tau$ and this tube to $p^{\prime}$ and replace $p$ by cl( $p-$ tube). Call this new collection $\hat{P}_{2,1}$ and call the induced function $\hat{T}_{2,1}$. Repeat this alteration using the triple $T_{2,1}: \hat{P}_{2,1} \rightarrow t(L)$ to get another triple $\hat{T}_{2,2}: \hat{P}_{2,2} \rightarrow t(L)$. Continue to make this alteration until, let's say, after $q$ times, $\hat{P}_{2, q}^{*}=M$. Let $T: P \rightarrow t(L)$ be this final triple and let $K$ be a subdivision of $K^{\prime}$ refining the sets in $P$. This completes the proof of (2.1).

(2.9) Remark. Suppose we stipulate that in performing the alteration in (2.8) if $\tau, \tau^{\prime} \in t\left(K^{\prime}\right)$ and $\tau \subseteq N_{u}, \tau^{\prime} \subseteq N_{u^{\prime}}$ and $u \neq u^{\prime}$, then the tubes used to join $\tau$ and $\tau^{\prime}$ to the appropriate sets in $\hat{P}_{2}$ are to be disjoint. We can then conclude that if $\tau \in t(K), \tau \subseteq p \in P$ and $\tau \subseteq \hat{p} \in \hat{P}$, then there exists $u, u^{\prime} \in U$ such that $T(p) \cup \hat{T}(\hat{p}) \subseteq \operatorname{st}\left(\Gamma_{u}\left(D^{2}\right), L\right)^{*} \cup \operatorname{st}\left(\Gamma_{u^{\prime}}\left(D^{2}\right), L\right)^{*}$. Although this remark need not concern us at the moment, it will be needed in the proof of Corollary (3.7.1).

Proof of Theorem (2.0). What remains to be done is to carefully study the proof of Proposition (2.1) in order to produce a homotopy from $F_{1}$ (which is already homotopic to $f$ ) to the monotone mapping obtained from the triple $T: P \rightarrow t(L)$.

(2.10) Let us assume that $L$ was chosen so that each of the sets $\operatorname{st}^{4}(\sigma, t(L))^{*}$, $\sigma \in t(L)$, has diameter less than $\epsilon$, where $\epsilon$ is chosen as follows. View $Q$ as embedded as a retract of an open subset of some Euclidean space. Use the locally convex structure of the open set and use the retraction to find $\epsilon>0$ such that 
any pair of mappings $h, g: X \rightarrow Q$ with $d(h(x), g(x))<\epsilon, x \in X$, are homotopic.

(2.11) For each $u \in U$, define a mapping $h_{u}: N_{u} \rightarrow D^{2}$ as follows. Let $h_{u}=g_{u}$ on $\operatorname{bd}\left(N_{u}\right)$ and let $h_{u}\left(\{x\} \times D^{m-1}\right)=h_{u}\left(\{x\} \times \dot{D}^{m-1}\right)$ for $x \in \alpha_{u}(I)$. Let $\hat{M}=\hat{P}_{1}^{*}$ and use (1.1) to obtain a mapping $\hat{F}$ from $\hat{M}$ to $Q$ which agrees with $\Omega_{u} \circ h_{u}$ (hence $\Omega_{u} \circ g_{u}$ ) on $\operatorname{bd}\left(N_{u}\right)$ for each $u \in U$. Let $F_{2}: M \rightarrow Q$ (respectively, $F_{3}: M \rightarrow Q$ ) be the extension of $\hat{F}$ by the $\Omega_{u} \circ h_{u}$ 's (respectively, $\Omega_{u} \circ$ $g_{u}$ 's).

In view of (2.10), $F_{1}$ and $F_{2}$ are homotopic. We can produce a homotopy $G$ from $F_{2}$ to $F_{3}$ as follows. On $\hat{M} \times I$ let $G=F_{2} \times \operatorname{id}_{I}\left(=F_{3} \times \operatorname{id}_{I}\right)$; let $G=$ $F_{2}$ on $M \times\{0\}$ and let $G=F_{3}$ on $M \times\{1\}$. Observe that $G=\Omega_{u} \circ s_{u}$ on the set $\left(\operatorname{bd}\left(N_{u}\right) \times I\right) \cup\left(N_{u} \times\{0,1\}\right)$ where $s_{u}=g_{u} \times \operatorname{id}_{I}$ on $\operatorname{bd}\left(N_{u}\right) \times I, s_{u}=g_{u}$ on $N_{u} \times\{1\}, s_{u}=h_{u}$ on $N_{u} \times\{0\}$. Extend $s_{u}$ to a mapping $S_{u}: N_{u} \times I \rightarrow D^{2}$ and let $G=\Omega_{u} \circ S_{u}$ on $N_{u} \times I$.

(2.12) Let $\hat{T}_{2,1}: \hat{P}_{2,1} \rightarrow t(L)$ be as in (2.7) and use (1.1) to obtain a mapping $\hat{F}_{3,1}$ from $\hat{P}_{2,1}^{*}$ agreeing with $F_{3}$ on $\operatorname{bd}\left(\hat{P}_{2,1}^{*}\right)$. Extend $\hat{F}_{3,1}$ to a mapping $F_{3,1}: M \rightarrow Q$ by letting $F_{3,1}=F_{3}$ on $M-\hat{P}_{2,1}^{*}$. In view of $(2.10), F_{3}$ and $F_{3,1}$ are homotopic. In a similar manner, using $\hat{T}_{2,2}: \hat{P}_{2,2} \rightarrow t(L)$, we obtain a mapping $F_{3,2}: M \rightarrow Q$; because of (2.10), $F_{3,2}$ and $F_{3,1}$ are homotopic. We continue to repeat this until we have $F_{3, q-1}$ homotopic to $F_{3, q}$; finally, $F_{3, q}$ is homotopic to the monotone mapping obtained in (1.4.1), this final homotopy also being guaranteed by (2.10).

3. In this section, we prove Theorem (3.0) below and use this theorem together with some ideas from the proof of Proposition (2.1) to obtain a result on approximating monotone mappings with monotone open mappings (see Corollary (3.7.1)).

(3.0) THEOREM. Let $M^{m}$ be a compact p.l. manifold $(m \geqslant 3)$ with triangulation $K$ and let $Q$ be a polyhedron with triangulation $L$. Let $P$ be a collection of nonempty subsets of $M$ with pairwise disjoint interiors, with each $p \in P$ a union of elements of $t(K)$, and with $P^{*}=M$. Let $T$ be a one-to-one function from $P$ onto $t(L)$ satisfying:

(3.0.1) $T\left(p_{1}\right) \cap \cdots \cap T\left(p_{q}\right) \neq \varnothing$ whenever $p_{1} \cap \cdots \cap p_{q} \neq \varnothing$.

(3.0.2) Each component of $T^{-1}\left(\sigma_{1}\right)$ meets $T^{-1}\left(\sigma_{2}\right)$ whenever $\sigma_{1} \cap \sigma_{2} \neq \varnothing$, $\sigma_{1}, \sigma_{2} \in t(L)$.

Then there is an open mapping $f$ from $M$ onto $Q$ such that the number of components of $f^{-1}(y)$ is equal to the number of components of $\{p \mid p \in P$ and $y \in T(p)\}^{*}$ and such that $f(x) \in \mathrm{st}^{2}(T(p), t(L))^{*}$ for $x \in p \in P$.

The proof of Theorem (3.0) is an immediate consequence of the following proposition (and its proof) and Proposition 3 in [18]. The observant reader will 
notice that the proof of (3.1) uses techniques developed by D. Wilson in [18] and [19]. As in (1.3), the collection of components of the sets in $P$ can be made simple; we assume that this has been done.

(3.1) Proposition. Assume the hypotheses of Theorem (3.0); then there are two sequences of finite collections of polyhedra, $\left\{J_{n}\right\}_{n=1}^{\infty}$ and $\left\{K_{n}\right\}_{n=1}^{\infty}$, satisfying:

(3.1.1) $J_{1}=t(L)$ and $K_{1}=P$; for $n \geqslant 2, J_{n}=\beta^{l_{n} L}$ where $l_{n}$ is a positive integer with $l_{n+1}>l_{n}$; the collection of components of the sets in $K_{n}$ is simple and $K_{n}^{*}=M$.

(3.1.2) For each $n \geqslant 2$, there is an integer $L_{n}$ with the property that if $j_{n} \in J_{n}$ and $j_{n-1}^{1}, \ldots, j_{n-1}^{r}$ are all the members of $J_{n-1}$ meeting st ${ }^{L_{n}+1}\left(j_{n}, J_{n}\right)^{*}$, then $j_{n-1}^{1} \cap \cdots \cap j_{n-1}^{r} \neq \varnothing$.

(3.1.3) There is a one-to-one function $R_{n}$ from $J_{n}$ onto $K_{n}$ with $R_{n}\left(j_{n}\right) \cap$ $R_{n}\left(j_{n}^{\prime}\right) \neq \varnothing$ whenever $j_{n} \cap j_{n}^{\prime} \neq \varnothing ;$ and $R_{n}^{-1}\left(k_{n}^{1}\right) \cap \cdots \cap R_{n}^{-1}\left(k_{n}^{r}\right) \neq \varnothing$ whenever $k_{n}^{1} \cap \cdots \cap k_{n}^{r} \neq \varnothing$. Also, if $k_{n} \in K_{n}$ and $k_{n-1} \in K_{n-1}$ with $k_{n} \cap k_{n-1} \neq \varnothing$, then $k_{n} \cap \operatorname{int}\left(k_{n-1}\right) \neq \varnothing$.

(3.1.4) For each $n \geqslant 2$, if $j_{n} \in J_{n}$ and $j_{n-1} \in J_{n-1}$, then $R_{n}\left(j_{n}\right) \cap$ $R_{n-1}\left(j_{n-1}\right) \neq \varnothing$ if and only if $j_{n-1}$ meets st $^{L_{n}}\left(j_{n}, J_{n}\right)^{*}$.

(3.1.5) If $j_{n} \cap j_{n}^{\prime} \neq \varnothing$, then $R_{n}\left(j_{n}\right) \subseteq N_{8 / 2^{n-1}}\left(R_{n}\left(j_{n}^{\prime}\right)\right)$.

(3.1.6) If $j_{n} \in J_{n}$ and $j_{n-1} \in J_{n-1}$ and $j_{n} \cap j_{n-1} \neq \varnothing$, then $R_{n-1}\left(j_{n-1}\right)$ $\subseteq N_{4 / 2^{n-1}}\left(R_{n}\left(j_{n}\right)\right)$.

(3.1.7) For each $j_{n} \in J_{n}$, there is a collection $A_{j_{n}}$ of polyhedral $m$ cells with the mesh of $A_{j_{n}}$ less than $4 / 2^{n}$, with $A_{j_{n}}^{*} \subseteq R_{n}\left(j_{n}\right)$, and with each component of $R_{n}\left(j_{n}\right)$ containing exactly one member of $A_{j_{n}}$. If $j_{n} \neq j_{n}^{\prime}$ and $j_{n} \cap j_{n} \neq \varnothing$, then $R_{n}\left(j_{n}^{\prime}\right) \cap$ a contains an $m-1$ cell for each $a \in A_{j_{n}}$. The various sets in the $A_{j_{n}}$ 's are pairwise disjoint.

Proof. We shall use (3.1.• $)^{n}$ to indicate condition (3.1.•) for the $n$th stage. Let $R_{1}=T^{-1}$ and for each $j_{1} \in J_{1}$ let $A_{j_{1}}$ be a collection of $m$ cells satisfying (3.1.7) $)^{1}$ where we assume that the metric on $M$ is such that the "metric conditions" in (3.1.5) $)^{1}$ and (3.1.6) $)^{1}$ are satisfied. Assuming that the construction has been done through the $n$th stage, we proceed to the $(n+1)$ st stage.

(3.2) Let $H$ be a triangulation of $M$ which subdivides each of the sets in $K_{n}$ and in the $A_{j_{n}}$ 's and which has mesh less than $4 / 2^{n}$. For each $y \in Q$, let $\epsilon_{y}>0$ with $N_{\epsilon_{y}}(y) \subseteq \operatorname{st}\left(y, J_{n}\right)^{*}$; let $\epsilon^{\prime}>0$ be such that every set of diameter less than $\epsilon^{\prime}$ is contained in some $N_{\epsilon_{y}}(y)$. Define $t\left(H \mid j_{n}\right)=\left\{\sigma \in t(H) \mid \sigma \subseteq R_{n}\left(j_{n}\right)\right\}$. Let $L$ be an integer such that $\mathrm{ST}^{L}\left(A_{j_{n}}^{*}, t\left(H \mid j_{n}\right)\right)^{*}=R_{n}\left(j_{n}\right)$ for each $j_{n} \in J_{n}$ and let $L_{n+1}=L+1$. Let $\epsilon>0$ with $\epsilon<\epsilon^{\prime} /\left(2 L_{n+1}+3\right)$ and let $J_{n+1}=\beta^{l n+1} L$ where $l_{n+1}>l_{n}$ is such that the mesh of $J_{n+1}$ is less than $\epsilon$ (this guarantees 
that (3.1.2) $)^{n+1}$ will hold) and such that st $^{L_{n+1}}\left(j_{n}, J_{n+1}\right)^{*} \subseteq \operatorname{st}\left(j_{n}, \beta^{l_{n}+1} L\right)^{*}$; this last condition will guarantee the conclusion in (3.0) that $f(x) \in \mathrm{st}^{2}(T(p), t(L))^{*}$.

(3.3) The following rule determines the sets in $t(H)$ which each $R_{n+1}\left(j_{n+1}\right)$ is to meet: if $j_{n+1} \in \mathrm{st}^{r+1}\left(j_{n}, J_{n+1}\right)-\operatorname{st}^{r}\left(j_{n}, J_{n+1}\right)$, then $R_{n+1}\left(j_{n+1}\right)$ is to meet exactly those elements of $t(H)$ in $R_{n}\left(j_{n}\right)$ which are in $\mathrm{ST}^{L_{n+1}-r}\left(A_{j_{n}}^{*}, t\left(H \mid j_{n}\right)\right)$. This basic rule guarantees that (3.1.4) $)^{n+1}$ will hold and this rule together with $(3.1 .7)^{n}$ guarantees that (3.1.5) ${ }^{n+1}$ and (3.1.6) $)^{n+1}$ will hold.

(3.4) We are now ready to begin the construction of the triple $R_{n+1}: J_{n+1}$ $\rightarrow K_{n+1}$. By using $l_{n+1}-l_{n}$ applications of the construction in (1.3), construct a triple $\hat{T}: \hat{P} \rightarrow J_{n+1}$ satisfying:

(3.4.1) The collection of components of the sets in $\hat{P}$ is simple and $\hat{T}\left(p_{1}\right) \cap$ $\cdots \cap \hat{T}\left(p_{q}\right) \neq \varnothing$ whenever $p_{1} \cap \cdots \cap p_{q} \neq \varnothing, p_{i} \in \hat{P}$.

(3.4.2) If $j_{n+1} \subseteq j_{n}$ and $h, h^{\prime} \in t(H)$ with $h \cup h^{\prime} \subseteq R_{n}\left(j_{n}\right)$ and $h \cap h^{\prime}$ an $m$ or $m-1$ face, then $\hat{T}^{-1}\left(j_{n+1}\right) \subseteq R_{n}\left(j_{n}\right)$ and $\hat{T}^{-1}\left(j_{n+1}\right) \cap \operatorname{int}\left(h \cup h^{\prime}\right)$ is connected.

(3.4.3) If $j_{n+1} \subseteq j_{n}, j_{n+1}^{\prime} \subseteq j_{n}^{\prime}$ with $j_{n+1} \cap j_{n+1}^{\prime} \neq \varnothing$ and $h \subseteq R_{n}\left(j_{n}\right)$, $h^{\prime} \subseteq R_{n}\left(j_{n}^{\prime}\right)$ with $h \cap h^{\prime}$ an $m$ or $m-1$ face, then

$$
\left(\hat{T}^{-1}\left(j_{n+1}\right) \cup \hat{T}^{-1}\left(j_{n+1}^{\prime}\right)\right) \cap \operatorname{int}\left(h \cup h^{\prime}\right)
$$

is connected (including the case $j_{n}=j_{n}^{\prime}$ ).

(3.5) Observe that if $j_{n+1} \subseteq j_{n}$, then $\hat{T}^{-1}\left(j_{n+1}\right)$ meets all members of $t(H)$ in $R_{n}\left(j_{n}\right)$ which $R_{n+1}\left(j_{n+1}\right)$ is to meet. We will now alter the $\hat{T}^{-1}\left(j_{n+1}\right)$ 's so that, for each $j_{n+1} \in J_{n+1}, \hat{T}^{-1}\left(j_{n+1}\right)$ will meet every member of $t(H)$ which $R_{n+1}\left(j_{n+1}\right)$ is to meet (see (3.3)). In particular, if $j_{n+1}^{r} \in \mathrm{st}^{r+1}\left(j_{n}, J_{n+1}\right)-$ st $^{r}\left(j_{n}, J_{n+1}\right)$ and $a \in A_{j_{n}}$, then the set $Z\left[j_{n}, a, j_{n+1}^{r}\right]$ (constructed below) meets every set in $\operatorname{ST}^{L+1-r}\left(a, t\left(H \mid j_{n}\right)\right)$. After adding each of the sets $Z\left[j_{n}, a, j_{n+1}^{r}\right]$, $a \in A_{j_{n}}$, to $\hat{T}^{-1}\left(j_{n+1}\right)$, we will have that $\hat{T}^{-1}\left(j_{n+1}\right)$ meets every set in $t\left(H \mid j_{n}\right)$ which $R_{n+1}\left(j_{n+1}\right)$ is to meet. For each $j_{n} \in J_{n}$ and for each $a \in A_{j_{n}}$, make the following alterations (all sets used in these alterations are to be in $\hat{K}^{n}-\hat{K}^{m-2}$ ).

(3.5.1) If $j_{n+1}^{1} \in \mathrm{st}^{1}\left(j_{n}, J_{n+1}\right)-\mathrm{st}^{0}\left(j_{n}, J_{n+1}\right)$, then form the set $Z\left[j_{n}, a, j_{n+1}^{1}\right]$ as follows. Choose $j_{n+1} \in \mathrm{st}^{0}\left(j_{n}, J_{n+1}\right)$ with $j_{n+1} \cap j_{n+1}^{1}=$ $j_{n} \cap j_{n+1}^{1}$ (for what follows, it would suffice that $j_{n+1} \cap j_{n+1}^{1} \neq \varnothing$, however, the stronger constraint will be needed in (3.6)). For each $h \in \mathrm{ST}^{L_{n+1}}\left(a, t\left(H \mid j_{n}\right)\right)$, $Z\left[j_{n}, a, j_{n+1}^{1}\right]$ is to contain an $m$ cell $b_{h}$ in $\operatorname{int}\left(h \cap \hat{T}^{-1}\left(j_{n+1}\right)\right)$; for each pair $h, h^{\prime} \in \mathrm{ST}^{L_{n+1}}\left(a, t\left(H \mid j_{n}\right)\right)$ with $h \cap h^{\prime}$ an $m-1$ cell, $Z\left[j_{n}, a, j_{n+1}^{1}\right]$ is to con. tain a tube in $\operatorname{int}\left(h \cup h^{\prime}\right) \cap \operatorname{int}\left(\hat{T}^{-1}\left(j_{n+1}\right)\right)$ connecting $b_{h}$ to $b_{h}^{\prime}$. Finally choose $\bar{h} \subseteq a$ and $\hat{h} \subseteq R_{n}\left(j_{n}^{1}\right)$, where $j_{n+1}^{1} \subseteq j_{n}^{1}$, with $\bar{h} \cap \hat{h}$ an $m-1$ face; $Z\left[j_{n}, a, j_{n+1}^{1}\right]$ is to contain a tube in $\operatorname{int}\left((\bar{h} \cup \hat{h}) \cap \hat{T}^{-1}\left(j_{n+1}\right)\right)$ connecting $b_{\bar{h}}$ to $\hat{T}^{-1}\left(j_{n+1}^{1}\right)$; conditions $(3.1 .7)^{n}$ and (3.4.3) guarantee that this is possible. Add $Z\left[j_{n}, a, j_{n+1}^{1}\right]$ 
to $\hat{T}^{-1}\left(j_{n+1}^{1}\right)$ and replace $\hat{T}^{-1}\left(j_{n+1}\right)$ with $\operatorname{cl}\left(\hat{T}^{-1}\left(j_{n+1}\right)-Z\left[j_{n}, a, j_{n+1}^{1}\right]\right)$. (3.5.2) If $j_{n+1}^{2} \in \operatorname{st}^{2}\left(j_{n}, J_{n+1}\right)-\operatorname{st}^{1}\left(j_{n}, J_{n+1}\right)$, then form the set $Z\left[j_{n}, a, j_{n+1}^{2}\right]$ as follows. Choose $j_{n}^{2}$ with $j_{n+1}^{2} \subseteq j_{n}^{2}$ and choose $j_{n+1}^{1} \in$ st $^{1}\left(j_{n}, J_{n+1}\right)$ with $j_{n+1}^{2} \cap j_{n+1}^{1} \neq \varnothing$ and $j_{n+1}^{2} \cup j_{n+1}^{1} \subseteq j_{n}^{2}$. For each $h \in$ $\mathrm{ST}^{L_{n+1}-1}\left(a, t\left(H \mid j_{n}\right)\right), Z\left[j_{n}, a, j_{n+1}^{2}\right]$ is to contain an $m$ cell $b_{h}$ in $\operatorname{int}\left(h \cap \hat{T}^{-1}\left(j_{n+1}^{1}\right)\right)$; for each pair $h, h^{\prime} \in \mathrm{ST}^{L_{n+1}-1}\left(a, t\left(H \mid j_{n}\right)\right)$ with $h \cap h^{\prime}$ an $m-1$ cell, $Z\left[j_{n}, a, j_{n+1}^{1}\right]$ is to contain a tube in $\operatorname{int}\left(h \cup h^{\prime}\right) \cap \operatorname{int}\left(\hat{T}^{-1}\left(j_{n+1}^{1}\right)\right)$ connecting $b_{h}$ to $b_{h^{\prime}}$. Choose the same $\bar{h} \subseteq a$ and $\hat{h} \subseteq R_{n}\left(j_{n}^{2}\right)$ which were used in constructing $Z\left[j_{n}, a, j_{n+1}^{1}\right] ; Z\left[j_{n}, a, j_{n+1}^{2}\right]$ is to contain a tube in $\operatorname{int}(\bar{h} \cup \hat{h})$ $\cap \hat{T}^{-1}\left(j_{n+1}^{1}\right)$ connecting $b_{\bar{n}}$ to $\hat{T}^{-1}\left(j_{n+1}^{2}\right)$. Add $Z\left[j_{n}, a, j_{n+1}^{2}\right]$ to $\hat{T}^{-1}\left(j_{n+1}^{2}\right)$ and replace $\hat{T}^{-1}\left(j_{n+1}^{1}\right)$ with $\mathrm{cl}\left(\hat{T}^{-1}\left(j_{n+1}^{1}\right)-Z\left[j_{n}, a, j_{n+1}^{2}\right]\right)$.

(3.5.3) For $r=2,3, \ldots, L_{n+1}$, inductively construct the sets $Z\left[j_{n}, a, j_{n+1}^{r}\right]$ for each $j_{n+1}^{r} \in \mathrm{st}^{r+1}\left(j_{n}, J_{n+1}\right)-\mathrm{st}^{r}\left(j_{n}, J_{n+1}\right)$ as in (3.5.2) and use them to alter the $\hat{T}^{-1}\left(j_{n+1}^{r}\right)$ 's.

After these alterations have been made for each $j_{n} \in J_{n}$ and each $a \in A_{j_{n}}$, we will have that $\hat{T}^{-1}\left(j_{n+1}\right)$ meets every member of $t(H)$ which $R_{n+1}\left(j_{n+1}\right)$ is to meet.

(3.6) For each $j_{n+1}^{\prime} \in J_{n+1}, j_{n+1}^{\prime} \subseteq j_{n}$, and for each $a \in A_{j_{n}}$, let $a^{\prime}$ be an $m$ cell of diameter less than $4 / 2^{n+1}$ in $\operatorname{int}\left(a \cap \hat{T}^{-1}\left(j_{n+1}^{\prime}\right)\right)$. If $j_{n+1}^{\prime \prime} \cap j_{n+1}^{\prime} \neq \varnothing$ and $j_{n+1}^{\prime \prime} \subseteq j_{n}$, then run a tube in $\operatorname{int}\left(a \cap\left(\hat{T}^{-1}\left(j_{n+1}^{\prime}\right) \cup \hat{T}^{-1}\left(j_{n+1}^{\prime \prime}\right)\right)\right)$ connecting $\hat{T}^{-1}\left(j_{n+1}^{\prime \prime}\right)$ to $a^{\prime}$; add the tube to $\hat{T}^{-1}\left(j_{n+1}^{\prime \prime}\right)$. If $j_{n+1}^{\prime \prime} \cap j_{n+1}^{\prime} \neq \varnothing$ but $j_{n+1}^{\prime \prime} \nsubseteq j_{n}$, then let $j_{n+1} \subseteq j_{n}$ be the set chosen in (3.5.1) to form $Z\left[j_{n}, a, j_{n+1}^{\prime \prime}\right]$; then $j_{n+1}^{\prime \prime} \cap j_{n+1}^{\prime} \cap j_{n+1} \neq \varnothing$ (see the parenthetical remark in (3.5.1)). Run a tube in int $\left(a \cap\left(\hat{T}^{-1}\left(j_{n+1}\right) \cup \hat{T}^{-1}\left(j_{n+1}^{\prime}\right) \cup \hat{T}^{-1}\left(j_{n+1}^{\prime \prime}\right)\right)\right)$ connecting $\hat{T}^{-1}\left(j_{n+1}^{\prime \prime}\right)$ to $a^{\prime}$; add the tube to $\hat{T}^{-1}\left(j_{n+1}^{\prime \prime}\right)$.

Let $K_{n+1}$ be the collection of $\hat{T}^{-1}\left(j_{n+1}\right)$ 's after all the above alterations have been made, let $A_{j_{n+1}}$ be the collection of $m$ cells constructed in (3.6), and let $R_{n+1}=\hat{T}_{n+1}^{-1}$. We leave to the reader to verify that $R_{n+1}: J_{n+1} \rightarrow K_{n+1}$ satisfies the necessary properties; the key to the verifications is the rule stated in (3.3).

(3.7) Observe that if, in addition to the hypotheses of Theorem (3.0), each set in $P$ is connected, then the open mapping $f$ obtained will also be monotone. This observation leads to the following

(3.7.1) COROLlaRY. Let $M^{m}$ be a compact, connected p. $l$. manifold with $m \geqslant 3$ and let $Q$ be a compact polyhedron. If $f$ is a monotone mapping of $M$ onto $Q$ and $\epsilon>0$, then there is a monotone open mapping $g$ of $M$ onto $Q$ with $d(f(x), g(x))<\epsilon$ for $x \in M$.

Proof. Let $L$ be a triangulation of $Q$ with the diameter of $\operatorname{st}^{11}(\sigma, t(L))$ 
less than $\epsilon$ and with $\operatorname{st}^{3}(\sigma, t(L))^{*}$ contractible for each $\sigma \in t(L)$. Let $\delta>0$ be such that the $3 \delta$ neighborhood of $\operatorname{st}^{i}(\sigma, t(L))^{*}$ is contained in $\operatorname{int}\left(\mathrm{st}^{i+1}(\sigma, L)^{*}\right)$ for $\sigma \in t(L)$ and $i=1,2$. Let $h$ be a mapping from $M$ to $Q$ with $h \delta$ :approximating $f$, with $h$ linearly mapping each simplex of $K$ (a triangulation of $M$ ) into a simplex of $L$, and with $h(M)$ meeting int $(\sigma)$ for each $\sigma \in t(L)$. Form a collection $\hat{P}$, a function $\hat{T}$ from $\hat{P}$ onto $t(L)$, and a subdivision $\hat{K}$ of $K$ as in (2.2); when making the collection of components simple, use "sufficiently small" tubes so that $h(p) \cup f(p) \subseteq N_{\delta}(\hat{T}(p))$ for each $p \in \hat{P}$.

If $\sigma, \sigma^{\prime} \in t(L)$ with $\sigma \cap \sigma^{\prime} \neq \varnothing$ and $U_{\sigma}=f^{-1}\left(\operatorname{int}\left(\operatorname{st}^{2}(\sigma, t(L))\right)^{*}\right)$ ), then $U_{\sigma}$ is an open connected set, $\hat{T}^{-1}(\sigma) \cup \hat{T}^{-1}\left(\sigma^{\prime}\right) \subseteq U_{\sigma}$; for $\tau \in t(K)$ with $\tau \cap U_{\sigma} \neq \varnothing$, $h(\tau) \subseteq \operatorname{st}^{3}(\sigma, t(L))^{*}$. Using arcs in $U_{\sigma} \cap\left(\hat{K}-\hat{K}^{m-2}\right)$ and using the simple connectivity of $\operatorname{st}^{3}(\sigma, t(L))^{*}$ for various $\sigma \in t(L)$, the techniques in the proof of Proposition (2.1) can be used to alter the triple $\hat{T}: \hat{P} \rightarrow t(L)$ and obtain a triple $T$ : $P \rightarrow t(L)$ satisfying the conditions of Theorem (3.0) with the sets in $P$ connected. Using Remark (2.9), it follows that if $x \in p^{\prime} \in \hat{P}$ before the alterations and $x \in$ $p \in P$ after the alterations, then $T(p) \subseteq \mathrm{st}^{7}\left(\hat{T}\left(p^{\prime}\right), t(L)\right)^{*}$. The corollary now follows from Theorem (3.0).

The next corollary is a direct consequence of Corollary (3.7.1) and Theorem (2.0).

(3.7.2) Corollary. A mapping from a compact and connected $p . l$. manifold $M^{m}(m \geqslant 3)$ to a connected polyhedron $Q$ is homotopic to a monotone open mapping of $M$ onto $Q$ if and only if $f_{*}: \pi_{1}(M) \rightarrow \pi_{1}(Q)$ is surjective.

This corollary contains the first published proof of the fact that if $n \geqslant 3$ and $m \geqslant 2$, then there is a monotone open mapping of the $n$-sphere $S^{n}$ onto the $m$-sphere $S^{m}$. This result was announced by R. D. Anderson in [1].

\section{REFERENCES}

1. R. D. Anderson, Open mappings of compact continua, Proc. Nat. Acad. Sci. U.S. A. 42 (1956), 347-349. MR 17, 1230.

2. S. Armentrout, Concerning cellular decompositions of 3-manifolds that yield 3-manifolds, Trans. Amer. Math. Soc. 133 (1968), 307-332. MR 37 \#5859.

3. S. Eilenberg, On the problems of topology, Ann. of Math. (2) 50 (1949), 247-260. MR 10, 726.

4. E. E. Floyd and F. B. Jones, Gordon T. Whyburn, 1904-1969, Bull. Amer. Math. Soc. 77 (1971), 57-72. MR 42 \#1639.

5. J. G. Hocking, Approximations to monotone mappings on non-compact two dimensional manifolds, Duke Math. J. 21 (1954), 639-651. MR 16, 158. \#2094.

6. J. F. P. Hudson, Piecewise linear topology, Benjamin, New York, 1969. MR 40

7. L. V. Keldyš, Monotone mappings of a cube onto a cube of higher dimension, Mat. Sb. 41 (83) (1957), 129-158. (Russian) MR 19, 874. 
8. L. V. Keldyš, Transformation of a monotone irreducible mapping into a monotoneinterior mapping and a monotone-interior mapping of the cube onto the cube of higher dimension, Dokl. Akad. Nauk SSSR 114 (1957), 472-475. (Russian) MR 19, 972.

9. - Examples of a monotone irreducible mapping of a three-dimensional cube onto a four-dimensional one, Dokl. Akad. Nauk SSSR 103 (1955), 957-960. (Russian) MR 17, 992.

10. A. Kolmogoroff, Über Offene Abbildungen, Ann. of Math. (2) 38 (1937), 36-38.

11. L. F. McAuley, Some fundamental theorems and problems related to monotone mappings, Proc. First Conf. on Monotone and Open Mappings (SUNY at Binghamton, Binghamton, N. Y., 1970), State Univ. of New York at Binghamton, Binghamton, N. Y., 1971, pp. 1-36. MR 44 \#4722.

12. - Open mappings and open problems, Topology Conference (Arizona State Univ., Tempe, Ariz., 1967), Arizona State Univ., Tempe, Ariz., 1968, pp. 184-202. MR 39 \#2134.

13. R. L. Moore, Concerning upper semi-continuous collections of continua, Trans. Amer. Math. Soc. 27 (1925), 416-428.

14. J. H. Roberts and N. E. Steenrod, Monotone transformations of 2-dimensional manifolds, Ann. of Math. (2) 39 (1938), 851-862.

15. S. Smale, $A$ Vietoris mapping theorem for homotopy, Proc. Amer. Math. Soc. 8 (1957), 604-610. MR 19, 302.

16. Stoilow, Sur les transformations continues et la topologie des fonctions analytiques, Ann. Sci. Ecole Norm. Sup. (3) 45 (1928), 347-382.

17. L. C. Siebenmann, Approximating cellular maps by homeomorphisms, Topology 11 (1972), 271-294. MR 45 \#4431.

18. D. C. Wilson, Open mappings of the universal curve onto continuous curves, Trans. Amer. Math. Soc. 168 (1972), 497-515. MR 45 \#7682.

19. - Open mappings on manifolds and a counterexample to the Whyburn conjecture, Duke Math. J. 40 (1973), 705-716. MR 47 \#9522.

20. - Monotone mappings of manifolds onto cells, Proc. First Conf. on Monotone and Open Mappings (SUNY at Binghamton, Binghamton, N. Y., 1970), State Univ. of New York at Binghamton, Binghamton, N. Y., 1971, pp. 37-54. MR 43 \#5501.

21. J.W.T. Youngs, Homeomorphic approximations to monotone mappings, Duke Math. J. 15 (1948), 87-94. MR 9, 524.

22. D. C. Wilson, On constructing monotone and $U V^{1}$ mappings of arbitrary degree, Duke Math. J. 41 (1974), 103-109.

\section{SCHOOL OF MATHEMATICS, INSTITUTE FOR ADVANCED STUDY, PRINCETON, NEW JERSEY 08540}

Current address: Department of Mathematics, University of Oklahoma, Norman, Oklahoma 73069 